\title{
Measurements of Charging of Apollo 17 Lunar Dust Grains by Electron Impact
}

\author{
Mian M. Abbas ${ }^{1}$, Dragana Tankosic ${ }^{2}$, \\ James F. Spann ${ }^{1}$, and Michael J. Dube ${ }^{3}$ \\ ${ }^{7}$ NASA-Marshall Space Flight Center, Huntsville, AL 35812 \\ ${ }^{2}$ University of Alabama in Huntsville, Huntsville, AL 35899 \\ ${ }^{3}$ NASA-Goddard Space Flight Center, Huntsville, AL 20771
}

\begin{abstract}
It is well known since the Apollo missions that the lunar surface is covered with a thick layer of micron size dust grains with unusually high adhesive characteristics. The dust grains observed to be levitated and transported on the lunar surface are believed to have a hazardous impact on the robotic and human missions to the Moon. The observed dust phenomena are attributed to the lunar dust being charged positively during the day by UV photoelectric emissions, and negatively during the night by the solar wind electrons. The current dust charging and the levitation models, however, do not fully explain the observed phenomena, with the uncertainty of dust charging processes and the equilibrium potentials of the individual dust grains. It is well recognized that the charging properties of individual dust grains are substantially different from those determined from measurements made on bulk materials that are currently available. An experimental facility has been developed in the Dusty Plasma Laboratory at MSFC for investigating the charging and optical properties of individual micron/sub-micron size positively or negatively charged dust grains by levitating them in an electrodynamic balance in simulated space environments. In this paper, we present the laboratory measurements on charging of Apollo 17 individual lunar dust grains by a low energy electron beam. The charging rates and the equilibrium potentials produced by direct electron impact and by secondary electron emission process are discussed.
\end{abstract}

Keywords. Lunar dust charging, electron impact charging of dust

PACS. 96.20,94.05.Bf, 95.30.wi, 96.12.Uv, 94.05.Sd, 94.20.wl

\section{INTRODUCTION}

The lunar surface, covered with a thick layer of fine micron/submicron dust grains, has formed by meteoritic impacts over a period of billions of years. Theoretical considerations and observational evidence acquired from the Apollo as well as subsequent lunar missions indicates the lunar surface and dust grains are electrostatically charged by the incident solar UV radiation and the solar wind plasma (e.g., Horanyi, et al., 1998; Stubbs, et al., Vondrak, et al., 2005). On the lunar dayside of the moon, the dust is believed to be charged positively by the photoelectric emissions with the incident solar UV radiation, and predominantly negatively by the incident solar wind electrons on the nightside There is considerable evidence to indicate that the charged fine lunar dust grains, smaller than a few microns in size, are levitated and transported to high altitudes and transported over long distances over the lunar terminator (e.g., McCoy et al., 1974; Rennilson et al., 1974; Pelizzari et al, 1978; Zook, 1991). The lunar dust with its toxic nature and high adhesive characteristics constitutes a major source of hazard for humans and the mechanical systems in the robotic and human exploration of the moon.

Although the basic principles and the underlying sources of the observed lunar dust phenomena are recognized, the extent and the details of the lunar dust charging, levitation, and transportation process remain poorly understood. The current theoretical models do not satisfactorily explain the observed lunar dust phenomena. A more definitive knowledge of the lunar dust phenomena with acquisition of the basic data is needed for engineering solutions and development of mitigating strategies. Whereas there is an urgent need for measurements of lunar dust density and size distribution on the near surface and high altitude environments, it is also necessary to acquire knowledge of the basic charging properties of lunar dust for evaluation of the dust grain charges and equilibrium potentials for development of dust mitigating strategies. Experimental results on the charging of individual lunar dust grains by photoelectric emissions have been presented in previous publications (Abbas et al., 2002, 2006, 2007). In this paper, we present some preliminary results on charging of lunar dust grains by low energy electrons to investigate the charging of lunar dust by the solar wind electrons on the lunar. The results presented are based only on the measured data, with the more detailed analysis to be discussed in subsequent publication with the availability of additional supporting data. 


\section{EXPERIMENTAL APPARATUS AND TECHNIQUE}

The measurement technique employed here is based on an experimental setup employing an electrodynamic balance (EDB) that permits levitation of submicron/micron size individual dust grains in simulated space environments. This technique has been used for measurements of charging of micron size dust grains by photoelectric emissions (Abbas et al., 2006, 2007), and by electron beams with energies in the keV range (e.g., Svestika, et al., 1993; Cermak, et al., 1995; Zilavy, et al., 1998). Individual dust grains, positively or negatively charged, are inserted in a trap placed in a small chamber where they are levitated in potential well formed by hemispherical top and bottom electrodes kept at DC potentials, and a ring electrode at an AC potential (Fig. 1).

Fig. 1. A schematic of the experimental setup based on an electrodynamic balance with an electron gun mounted at the top.

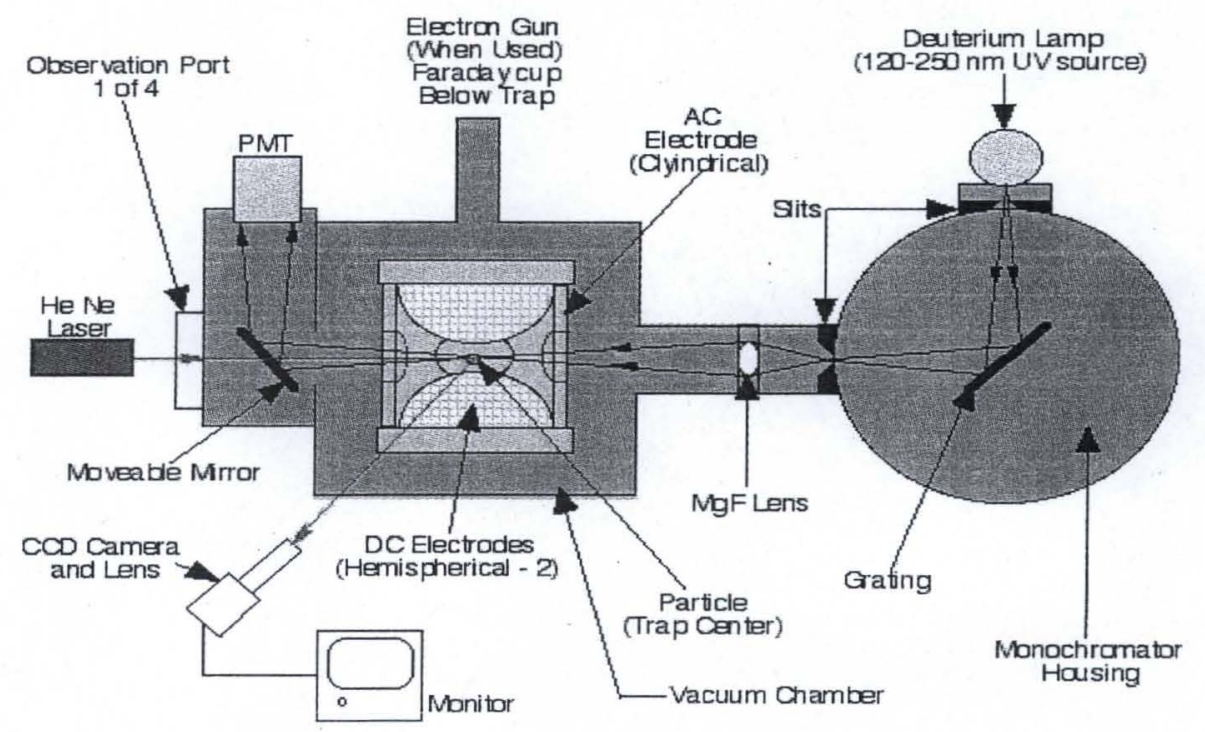

The experimental apparatus consists of the following: (1) The electrodynamic balance. (2) Computer controlled $\mathrm{DC}$ and $\mathrm{AC}$ power supplies (3) Optical equipment for imaging a levitated particle on a monitor. (4) An electron gun mounted at the top of the chamber with a power supply and controller for a selecting a mono-energetic Gaussian electron beam at desired energy levels. (5) A Faraday cup below the trap with an electrometer to measure the electron current. (6) Vacuum pumps to evacuate the system to pressures of $\sim 10^{-5}-10^{-6}$ torr.

Positively or negatively charged particles are stably trapped in the EDB and the system is evacuated to pressures of $\sim 1-5$ torr. The particle effective diameter is determined by using the marginal stability condition referred to as the "spring point method" (e.g., Davis, 1985; Spann et al., 2001; Abbas et al., 2002). The system is then evacuated to pressures of $\sim 10^{-5}-10^{-6}$ torr, and the levitated particle exposed to an electron beam at selected energy levels in the $25-100 \mathrm{eV}$ range. As the particle discharges or charges with the impact of electrons, the particle position deviates from the trap center, requiring an adjustment in the DC voltage and providing a direct measurement of the particle charge in accordance with,

$$
q(t)=\frac{g z_{o} m}{C_{o}} \frac{1}{V_{D C}(t)},
$$

where $q(t)$ and $V_{D C}(t)$ represent the particle charge and the corresponding DC electrode voltage as a function of time, $\mathrm{m}$ is the particle mass, $g$ is the gravitational acceleration, $C_{0}$ is a geometric constant of the trap experimentally determined to be 0.68 , and $z_{0}=0.765 \mathrm{~cm}$ is the DC electrode distance to the trap center. With measurements of $V_{D C}$, the effective particle diameter $\mathrm{D}$, the mass $\mathrm{m}$ is calculated from the predetermined lunar dust grain density $\rho=1.8$ $\mathrm{gcm}^{-3}$, the particle charge $q(t)$ is thus determined directly from equation (1) as a function of time.

Detailed descriptions of the experimental apparatus and the mathematical equations for evaluation of the measured quantities on an EDB have been given in previous publications (e.g., Davis, 1985; Spann et al. 2001; Abbas et al. 2003, 2005, 2006, 2007;). 


\section{MEASUREMENTS ON APOLLO 17 LUNAR DUST GAINS}

The measurements of charging of Apollo 17 lunar dust grains presented in this paper were made on particles of $\sim 0.2-8 \mu \mathrm{m}$ diameters by exposing them to mono-energetic electron beams at energies of $\sim 25-100 \mathrm{eV}$. As the particle charges or discharges by interaction with the incident electrons, the change in particle charge is determined as a function of time in accordance with equation (1). Some theoretical models for charging of individual small spherical models have been developed (e.g., Chow et al., 1994). In the following, we present the measurements based on charging/discharging of some selected particles focusing on the basic nature of the charging processes as a function the particle size, the electron energy, and the incident electron beam current. More detailed analytical results based on the experimental data presented in this paper will be discussed in subsequent publications.

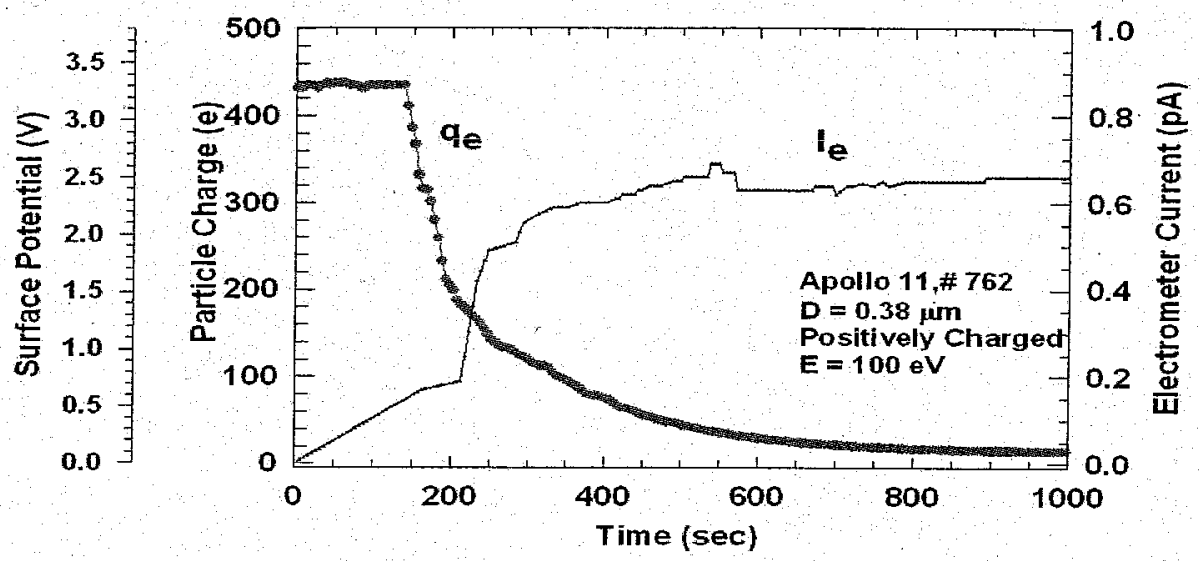

Fig. 2a. A $0.38 \mu \mathrm{m}$ positively charged Apollo 17 dust particle exposed to a $100 \mathrm{eV}$ electron beam, discharges from an initial charge and potential of: $\mathrm{a} \sim 430 \mathrm{e}$. and $\phi \sim 3.3 \mathrm{~V}$ to a low eauilibrium values: $\mathrm{a} \sim 14 \mathrm{e} . \phi_{.} \sim 0.1 \mathrm{~V}$.

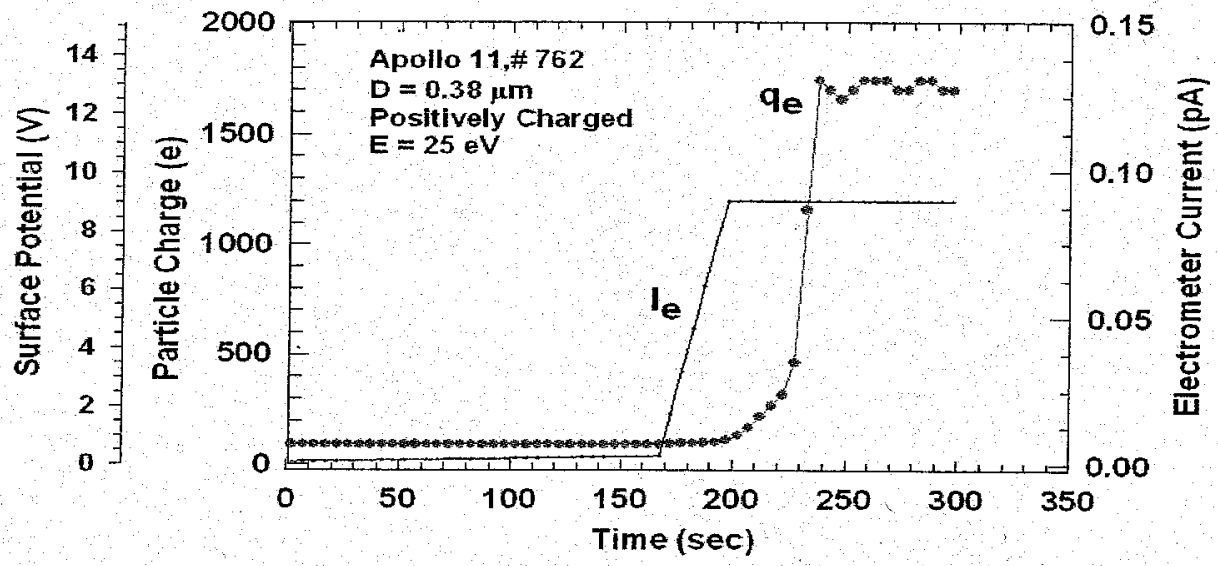

Fig. 2b. Re-charging of the particle in Fig. a, from an initial $\mathrm{q} \sim 80 \mathrm{e}$ to an equilibrium value of $\sim 1700 \mathrm{e}\left(\phi_{s} \sim 7\right.$ V), by exposing it to a 25 eV electron beam.

Figures $2 \mathrm{a}-\mathrm{b}$ represent the measurements of the charge $q(t)$ (in units of elementary electron charge e) on a positively charged $0.38 \mu \mathrm{m}$ Apollo 17 dust grain exposed to an electron beam with (a) Discharging with a $100 \mathrm{eV}$ beam from an charge of $\sim 430 \mathrm{e}$ to an equilibrium charge of $\sim 10 \mathrm{e}$, corresponding the particle surface potentials $\phi_{s} \sim$ 
3.2 to $0.1 \mathrm{~V}$, attained over a time period of $\sim 1000 \mathrm{sec}$. Also shown is the electron beam current $\mathrm{I}_{\mathrm{e}}$ (in picoAmperes $=10^{-12} \mathrm{~A}$ ) measured by an electrometer with a Faraday cup placed below the trap. The equilibrium potential represents the stage when the primary beam electrons sticking to the dust grain are equal to the induced secondary electrons ejected by the grain, with no net change in the charge. Figure $2 \mathrm{~b}$ shows the measurements on the same particle with an initial charge of $\sim 80 \mathrm{e}$ exposed to a $25 \mathrm{eV}$ electron beam. With an increase in the electron beam current, the particle is now observed to charge positively by induced secondary electron emissions to a final equilibrium charge of $\sim 1700 \mathrm{e}\left(\phi_{s} \sim 13 \mathrm{~V}\right)$

The discharging/charging processes of a $1.9 \mu \mathrm{m}$ positively charged Apollo 17 dust grain exposed to a $25 \mathrm{eV}$ electron beam are shown in Figs. 3a-c. Figure 3a shows discharging of the grain at relatively low electron currents to a near equilibrium potential of $\sim 1 \mathrm{~V}$. Figure $3 \mathrm{~b}$ represents a short discharging process of the same particle to an equilibrium level, followed by several phases of charging and equilibrium levels, with the last equilibrium potential at $\sim 1.8 \mathrm{~V}$. Each higher equilibrium level is reached with an increase in the secondary electron emissions induced by a higher level of the electron beam current as indicated by the $\mathrm{I}_{\mathrm{e}}$ plot on the figure. Figure $3 \mathrm{c}$ indicates similar processes of discharging/charging from an initial charge of $\sim 725 \mathrm{e}$ to an equilibrium level of $\sim 950 \mathrm{e}$.

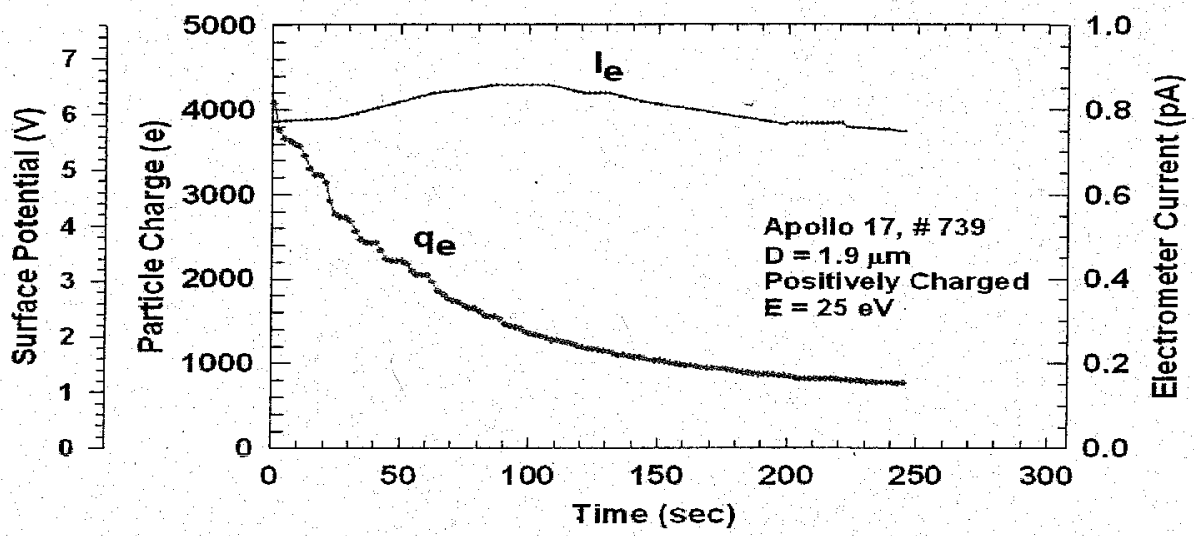

Fig. 3a. A $1.9 \mu \mathrm{m}$ positively charged Apollo 17 dust particle discharged by exposing it to a $25 \mathrm{eV}$ electron beam. The particle reaches an equilibrium potential $\phi_{n} \sim 1 \mathrm{~V}$. and does not discharge anv further.

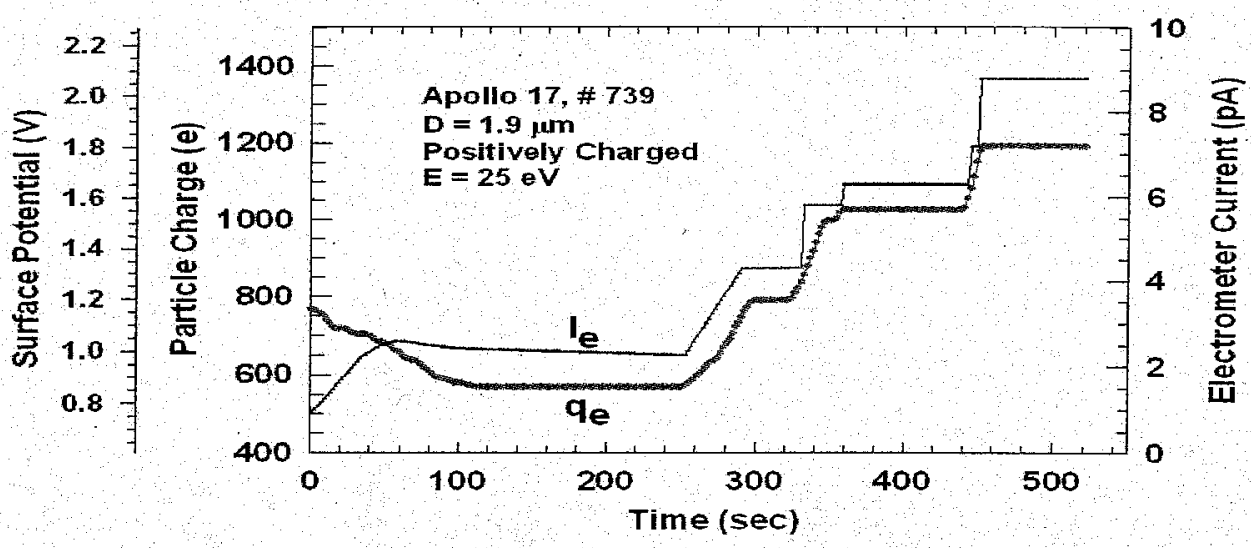

Fig. 3b. Charging of the particle in Fig. 3a, in a series of equilibrium values, of charge, by successively increasing the electron current. 


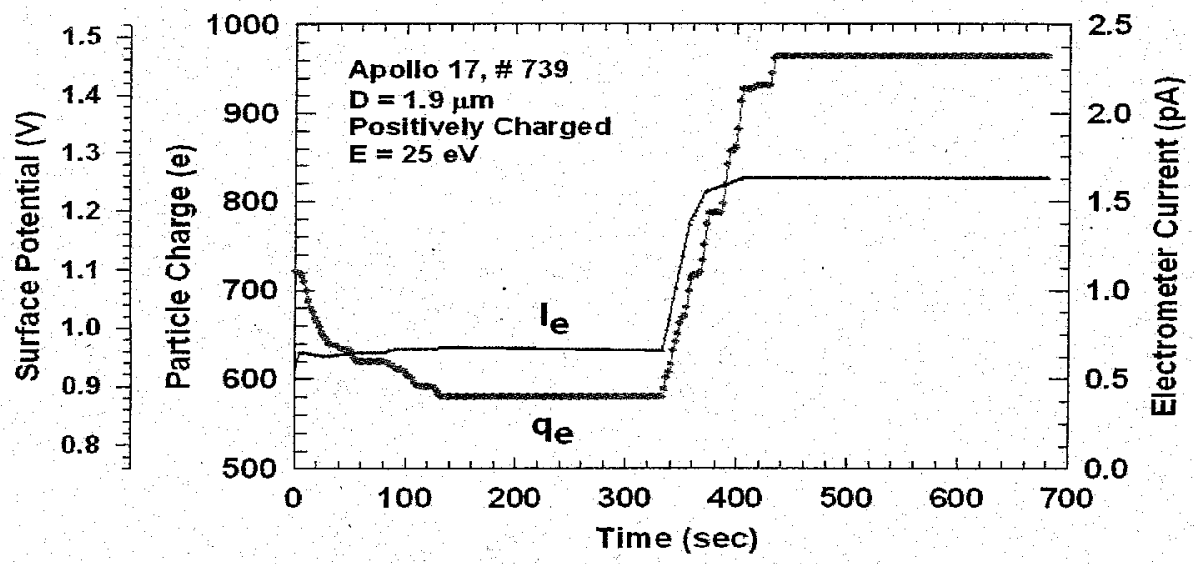

Fig. 3c. Charging of a $0.19 \mu \mathrm{m}$ positively charged Apollo 17 dust particle exposed to a $25 \mathrm{eV}$ electron beam. The particle reaches an eauilibrium potential at $a \sim 960 \mathrm{e} . \phi_{\sim} \sim 1.43 \mathrm{~V}$.

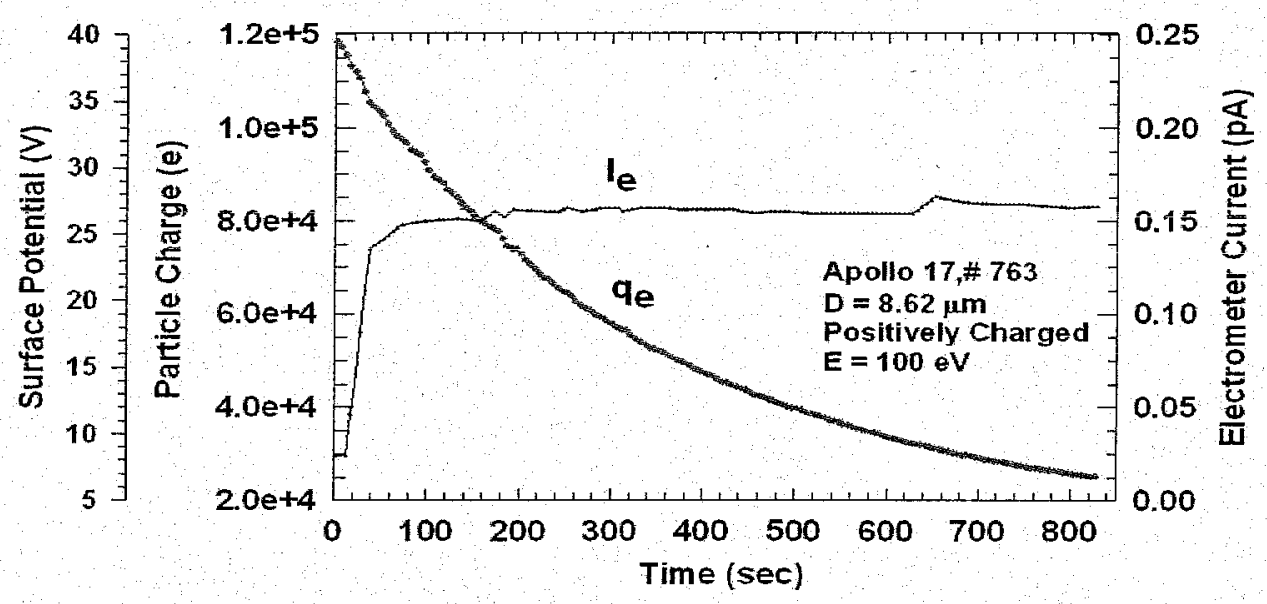

Fig. 4a. Discharging of a $8.62 \mu \mathrm{m}$ positively charged Apollo 17 dust particle exposed to a $100 \mathrm{eV}$ electron beam. The particle reaches a low equilibrium potential with $\mathrm{q} \sim 120,000 \mathrm{e}, \phi_{s} \sim 40 \mathrm{~V}$.

Figures 4a-b indicate the discharging/charging of a $8.62 \mu \mathrm{m}$ Apollo 17 positively charged lunar dust grain. Figure 4a shows the discharge from an initial charge of $\mathrm{q} \sim 120,000 \mathrm{e}\left(\phi_{s} \sim 40 \mathrm{~V}\right)$ to a near equilibrium charge of $\mathrm{q}$ $\sim 25,000 \mathrm{e}\left(\phi_{s} \sim 7 \mathrm{~V}\right)$ under exposure to a beam of $100 \mathrm{eV}$ electron with a relatively low current of $\sim 0.15 \mathrm{pA}$. In Fig. $4 \mathrm{~b}$, the same particle is exposed to a beam of $25 \mathrm{eV}$, and is observed to discharge at low currents of $\sim 0.1 \mathrm{pA}$, and positively to a higher values and potentials at much higher beam currents $(\sim 20-45 \mathrm{pA})$ with the secondary electron emissions dominating the primary electrons sticking to the grain. 


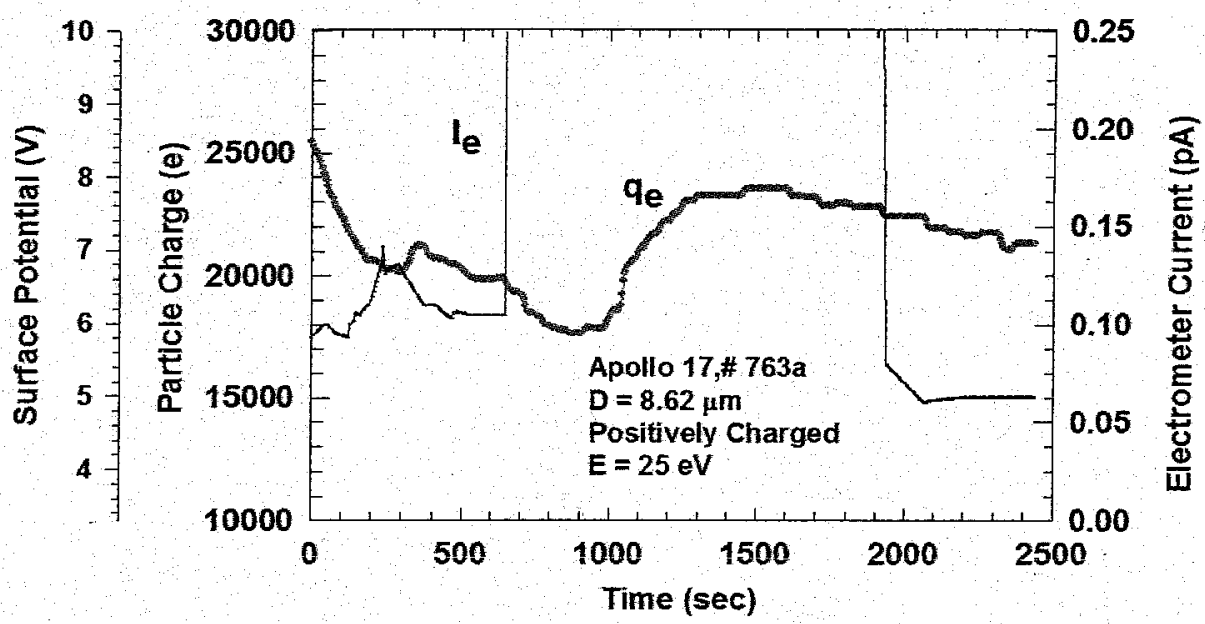

Fig. 4b. Same as in Fig. 4a, with the particle now exposed to a beam of $25 \mathrm{eV}$, and is observed to discharge at low currents of $\sim 0.1 \mathrm{pA}$, and positively to a higher values and potentials at much higher beam currents $(\sim 20-45 \mathrm{pA})$.

In the following, we present the measurements made on two negatively charged Apollo 17 dust grains exposed to electron beams at energies of $100 \mathrm{eV}$ and $25 \mathrm{eV}$. Figure 5a represents the discharging of an Apollo 17 negatively charged dust grain of $0.29 \mu \mathrm{m}$ size when exposed to a $25 \mathrm{eV}$ electron beam. The particle discharges from an initial charge of $\sim 200 \mathrm{e}\left(\phi_{s} \sim 2.0 \mathrm{~V}\right)$ to an equilibrium value of $\sim 22 \mathrm{e}\left(\phi_{s} \sim 0.2 \mathrm{~V}\right)$, representing the secondary electron emissions being balanced by the electron sticking process.

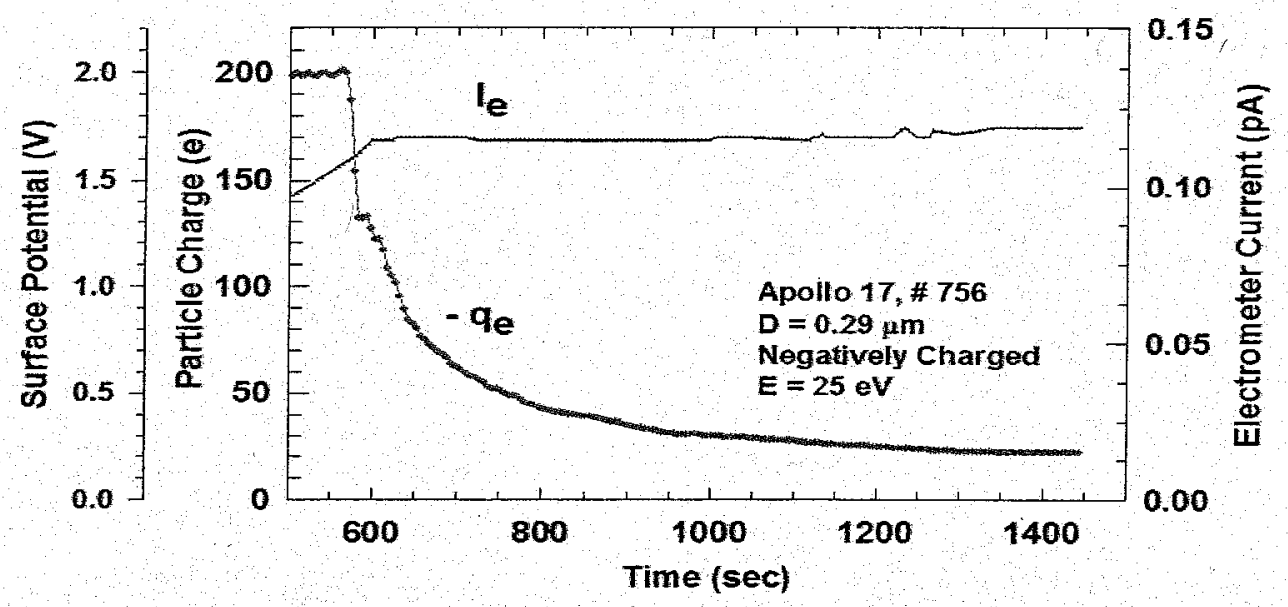

Fig. 5. A $0.29 \mu \mathrm{m}$ negatively charged Apollo 17 dust particle exposed to a 25 eV electron beam, discharges from the initial charge of $200 \mathrm{e}$ to equilibrium values of $\mathrm{q} \sim 22 \mathrm{e}, \phi_{s} \sim 0.2 \mathrm{~V}$. 
Figure 6a shows the discharging of a $0.35 \mu \mathrm{m}$ negatively charged dust grain exposed to a $25 \mathrm{eV}$ electron beam, when the beam current is raised to a sufficiently high value of $\sim 0.15 \mathrm{pA}$, and reaching an equilibrium value of $35 \mathrm{e}$ $\left(\phi_{s} \sim 0.3 \mathrm{~V}\right)$. As shown in Fig. $6 \mathrm{~b}$, when the electron current is steadily raised to a higher value, the negatively charged particle increase in charge to an equilibrium value of $\sim 45 \mathrm{e}$, representing an increase in the primary electrons sticking to the grains and balanced by the electron secondary electron emission from the particle.

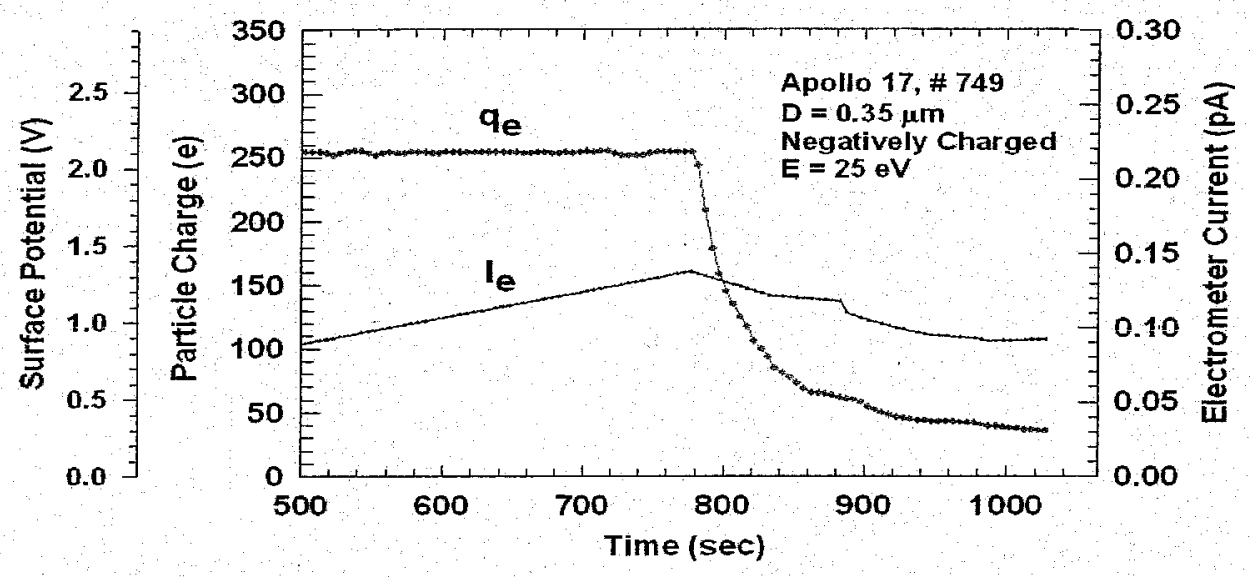

Fig. 6a A $0.35 \mu \mathrm{m}$ negatively charged Apollo 17 dust particle exposed to a $25 \mathrm{eV}$ electron beam discharged from the initial charged from the initial charge of $254 \mathrm{e}$ to the eauilibrium values of a $\sim 35 \mathrm{e} . d \sim 0.3 \mathrm{~V}$.

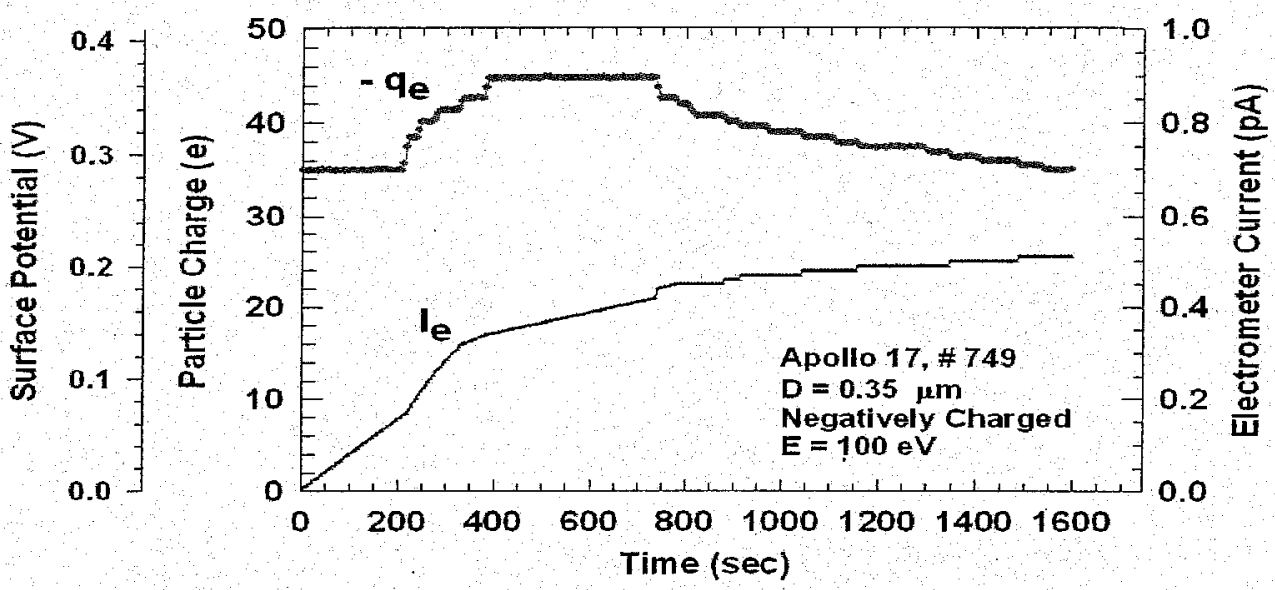

Fig. 6b. Same as in Fig.6a, with the beam electron current steadily raised to higher values. The particle charges to higher potentials, and then discharges to an equilibrium values of about the same as the initial $\mathrm{q} \sim 35 \mathrm{e}, \phi_{s} \sim 0.3 \mathrm{~V}$. 


\section{SUMMARY AND CONCLUSIONS}

Measurements of charging of individual micron size Apollo 17 dust grains levitated in an electrodynamic balance were made by exposing them to electron beams of $25-100 \mathrm{eV}$. Both positively and negatively charged particles in the 0.3 to $8.8 \mu \mathrm{m}$ size range were employed for measurements of the charging and discharging processes. The following conclusions may be drawn from the preliminary measurement presented here.

1. Positively charged particles larger than a few micron in size, discharge at a rapid rate when exposed to 25 $100 \mathrm{eV}$ electron beams, reaching equilibrium potentials indicating a balance between the sticking electrons and the secondary electron emissions.

2. Submicron size positively charged particles at low surface potentials generally charge more positively to higher charges when exposed to $25 \mathrm{eV}$ electron beams, indicating the secondary electron emission process dominating the primary electron sticking.

3. Equilibrium charges and surface potentials of dust grains are a function of the particle size, the incident electron density, with the equilibrium potentials depending on the beam current.

4. Negatively charged particles exposed to $25-100 \mathrm{eV}$ electron beams generally discharge to equilibrium potentials, indicating a balance between the secondary electron emission and the primary electron sticking at lower particle potentials.

5. More detailed analysis of the measured data with additional measurements will be discussed in subsequent publications.

\section{ACKNOWLEDGEMENTS}

The work reported has been supported the MSFC-IRAD and the NESC-Mechanical Systems Lunar Dust Assessment program. Helpful discussions with Drs. Dennis Gallagher Robert Sheldon and are gratefully acknowledged:

\section{REFERENCES}

Abbas, M. M., et al. Photoemission Experiments for Charge Characteristics of Individual Dust Grains, Physica Scripta, T98, 99103. 2002.

Abbas, M. M., et al., "Photoelectric Emission Measurements on the Analogs of Individual Cosmic Dust Grains", Astrophysical Journal, Vol. 645. No.1, July 1, 2006.

Cermak, I., E. Grun, and I. Svestika, "New results in the study of electric charging of dust particles, Adv. Space Res., Vol. 15, NO. $10,1059-1064,1995$.

Chow, V. W., D. A. Mendis, and M. Rosenberg, "Secondary emission from small dust grains at high electron energies", IEEE Trans. Plasma Sci., Vol. 22, No. 2, 1994.

Davis, E. J., "Electrodynamic balance stability characteristics and applications to the study of aerocolloidal particles", Langmuir, $1,379-387,1985$

Horanyi, M., et al., "Electrostatic charging properties of simulated lunar dust", Geophysical Research Letters, 22, 2079-2082, 1995.

Horanyi, M., et al., "Electrostatic charging properties of Apollo 17 lunar dust", Journal of Geophysical Research, 103, 8575$8580,1998$.

Johnson, S. W., et al. Environmental effects on lunar astronomical observatories, $2^{\text {nd }}$ Conf. On Lunar Bases \& Space Activities, $1,329-335,1992$.

McCoy, J.E., and Criswell, D.R., "Evidence for a high altitude distribution of lunar dust", Proc. $5^{\text {th }}$ Lunar Conference, 3, 2991$3005,1974$.

Pelizzari, M. A., and D. R. Criswell, "Lunar dust transport by photoelectric charging at sunset", Proc. Lunar Planet. Sci. Conf., $9^{\text {th }}, 3225-3237,1978$.

Rennilson, J. J., et al., "Surveyor observations of Lunar horizon-glow", The Moon, 10, 121-142, 1974.

Spann, J.F., et al., "Electrodynamic Balance for studies of cosmic dust particles”, Physica Scripta, T89, 147-153, 2001.

Stubbs, T.J., et al., "A dynamic fountain model for lunar dust", Advances in Space Research, 37(1), 59-66, 2006.

Svestika, J., I. Cermak, and E. Grun, "Electric charging and electrostatic fragmentation of dust particles in laboratory", Adv. Space Res., Vol. 13, NO. 10, 199-202, 1993.

Vondrak, R. R., et al., "A Dynamic Fountain Model for Dust in the Lunar Exosphere", Workshop on Dust in Planetary Systems, Kauai, HI, 2005. 
Zilavy, P., Z. Sternovsky, I. Cermak, Z. Nemecek, and Safrankova, "Surface potential of small particles charged by the mediumenergy electron beam", Vacuum, Vol. 50, No. 1-2, 139-142, 1998.

Zook, H. A., and McCoy, E., "Large scale lunar horizon glow and a high altitude lunar dust exosphere", Geophysical Research Letters, 18, 2117, 1991. 